\title{
Numerical analysis of the physical phenomena in the working zone in the rolling process of the round thread
}

\author{
L. Kukielka \& K. Kukielka \\ Department of Mechanical Engineering, \\ Koszalin University of Technology, Poland
}

\begin{abstract}
Thread rolling is a very complicated technological process. To improve the quality of the product and reduce production cost of the round thread, we should know the physical phenomena existing in the contact zone between rolls and deform work pieces. Therefore, this paper presents the physical and mathematical models of deformations (displacements and strains) and stress in the cold process of round thread rolling. The process is initially considered in a geometrically and physically non-linear regime, as well as a boundary value problem. The physical phenomena on a typical incremental step were described using a step-by-step incremental procedure, with an updated Lagrangian formulation. The state of strains was described by Green-Lagrange's tensor, while the state of stress by the second symmetrical Pioli-Kirchhoff's tensor. The object was treated as an elastic (in the reversible zone) and visco-plastic body (in the non-reversible zone) with mixed hardening. The variational equation of motion in three dimensions for this case was proposed. Then, the finite elements methods (FEM) and dynamic explicit method (DEM) were used to obtain the solution. The application is developed for the method of finite elements in the ANSYS programme, which provides a complex time analysis for displacement, strains and stresses occurring in the object. The effective discrete computable model which counts minimum degrees of freedom and a guide to convergence of solutions for the maximum value of stresses and strains, is proposed. Examples of simulation of the influence on various process conditions on the states of strain and stress are presented.
\end{abstract}

Keywords: round thread, rolling process, model investigation, equation of motion, FEM, ANSYS, numerical analysis, DEM, state of strain, state of stress. 


\section{Introduction}

The development of industry, especially in the areas of motorization and construction engineering, results in the fact that there are new requirements for products on a higher level. They require an improvement of the technological quality and an enlarged corrosion resistance. One of the methods of mechanical working is thread rolling, which gives a surface layer with profitable and usable properties. Using this modern technology gives basic advantages, such as an increase in the physical and mechanical properties of surface layer, higher wear resistance, enlarged dimension and shape accuracy of the screws and the increased efficiency of the thread rolling process. The properties beneficial for exploitation purposes are the result of plastic deformations in the rolled surface layer (fig. 1(b)).

Round threads with quick pitch make up the specific group. The screw joint folding from the nut and screw (fig. 1a) is used mainly in the construction of communication tunnels and construction engineering to support ceilings and in earth works for the protection of excavations. The screw threads are used with a nominal diameter of $d=31$ and $d=38 \mathrm{~mm}$, the pitch of $P=12,56$ and $P=12,78 \mathrm{~mm}$ respectively and typical lengths of $L=6000 \mathrm{~mm}$.

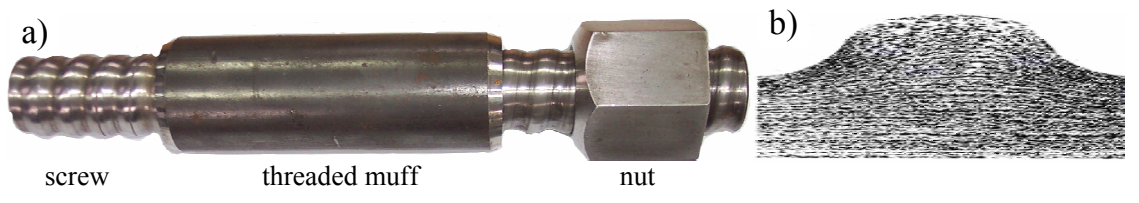

Figure 1: The screw joint (a) and the surface layer of the round thread after rolling (b).

The basic problems in designing the rolling process of the round thread on a pipe are elaborate proper construction of the tool (threading head) and selection of the processing condition for providing technical requirements and property of the surface layer of the screw, simultaneously increasing the tool life and process productivity. Presently, this technology is not used in Polish industry, with no base of scientific knowledge about this process or guidelines to the selection of process conditions. Therefore, at the Koszalin University of Technology, scientific research is working out the round thread rolling method on pipes and also the realization with high velocities and high temperatures.

\section{Introducing investigations}

The aim of the introductory investigations were to check the possibility of making the round thread on a pipe with a rolling method on a typical rolling mill and to determinate the important influential factors on the quality of the thread.

The research has shown that the thread rolling process on pipes is very complicated technological process. The influencing factors on the rolling process 
and the quality of screw can be divided into three principal groups: materials property, geometrical property of the thread and tool, and technological parameters of the rolling. Process characterizing is with the high instability, load of the pipe with low texturing stiffness, high force of working, causes that pose difficult problems to produce the screw with the expected quality.

Also, there is a big problem for each kind of material, dimension of pipe (outline diameter, partition gauge and accuracy dimensional - shape) and state of the surface layer (roughness, state of internal stresses), also selection of optimal working outline surface of the rolls, the kind of lubricate factor and the rolling factor (velocity of moving rolls, velocity of rolling, material and setting of support, force of pressure) to aim for a desirable quality of the thread and productivity of the process. Not satisfy these conditions can cause such defects as: pitting, irregular outline, crack, overlapping, incomplete outline, curving and dimensional deviation.

a)

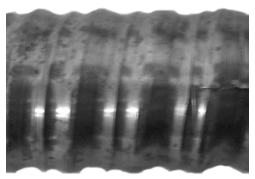

b)

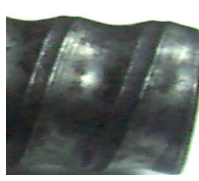

c)

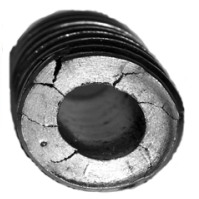

d)

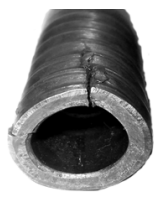

e)

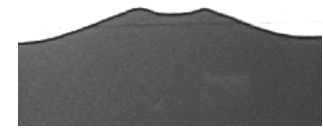

Figure 2: Defects formed by the thread rolling process: irregular outline (a), pitting (b), crack (c), overlapping (d), incomplete outline (e).

Achieving a high quality of thread requires a very precise manufacturing process together with the right design of the tool and other important factors, all of which have an influence on the final product.

Elaborate experimental knowledge of the rolling process and optimization with a high numbers of factors is a task that is time-consuming and makes expensive experimental research, where the theory of experiment planning must be applied. Therefore, the problem was approached using a theoretical model to elaborate the pipe rolling process, where the variational and the finite element method, were applied. Elaborating this model is necessary because of the complexity and the analysis of the physical phenomena for the real condition of the rolling process (geometry of the tools, values of the technological factors, the friction conditions, and so on). For this condition, known occurrences of the displacement, strain and stress is used to obtain the pressures and forces of the rolling, also to describe the properties of the thread surface.

The computational calculations were made with an elaborated application in ANSYS system. Exemplary results of numerical simulation concern the influence of friction conditions in the contact zone tool - work pieces (pipe steel C55) on the outline of thread and states of displacement, strains and stresses in the surface layer, for the thread rolling process on cold (nominal diameter $d=31 \mathrm{~mm}$ ). 


\section{Mathematical model of process}

A mathematical model of the process is formulated in increments and contains the following: a material model, an equation of motion and deformation, with initial and boundary conditions.

\subsection{Material model}

\subsubsection{Incremental model of yield stress}

Yield stress $\sigma_{y}$ is the most important parameter characterizing the resistance of a visco-plastic deformation. The incremental model of the yield stress for a typical step time $t \rightarrow \tau=t+\Delta t$ was defined as [2]:

$$
\Delta \sigma_{y}=F_{2}(y) \Delta y+\frac{\partial F_{1}[\bullet]}{\partial \dot{\varepsilon}_{e q}^{(V P)}} \Delta \dot{\varepsilon}_{e q}^{(V P)}+\frac{\partial F_{1}[\bullet]}{\partial \sigma_{s t}} F_{3}\left(\varepsilon_{e q}^{(V P)}\right) \Delta \varepsilon_{e q}^{(V P)},
$$

where $\Delta \varepsilon_{e q}^{(V P)}, \Delta \dot{\varepsilon}_{e q}^{(V P)}$ are the incremental of effective visco-plastic strain and strain rate, $F_{2}(y) \Delta y$ is the component of change in the initial yield stress with a change of chemical composition, $\left[\partial F_{1}[\bullet] / \partial \sigma_{s t}\right] F_{3}\left(\varepsilon_{e q}^{(V P)}\right) \Delta \varepsilon_{e q}^{(V P)}$ is the component of change in the temporary yield stress $\sigma_{y}$ with change of the visco-plastic strain, $\left[\partial F_{1}[\bullet] / \partial \dot{\varepsilon}_{e q}^{(V P)}\right] \Delta \dot{\varepsilon}_{e q}^{(V P)}$ is the component of change in the temporary yield stress with change of the visco-plastic strain rate, $\sigma_{s t}$ is the state stress depending on the accumulated effective visco-plastic strain and time.

\subsubsection{Elastic/visco-plastic material model}

A new model of mixed hardening for isotropic material which includes the combined effects of elasticity (a reversible domain), visco-plasticity (a non-reversible domain) $(E / V P)$ is used. The model takes into account the history of the material.

The constitutive equation of increment components of a total strain tensor takes form:

$$
\Delta \varepsilon_{i j}=\frac{1}{1-\widetilde{S}^{* *}}\left[D_{i j k l}^{(E)} \Delta \sigma_{k l}-A\right]
$$

and of increment components of the total stress tensor:

$$
\Delta \sigma_{i j}=C_{i j k l}^{(E)} \Delta \varepsilon_{k l}-\psi \widetilde{S}_{i j}^{*}\left[\widetilde{S}_{i j} C_{i j k l}^{(E)} \Delta \varepsilon_{k l}-A\right],
$$

where:

$$
\widetilde{S}^{* *}=\widetilde{S}_{i j}^{*} C_{i j m n}^{(E)} \widetilde{S}_{m n},
$$

is a positive scalar variable, 


$$
\tilde{S}_{i j}^{*}=\frac{\tilde{S}_{i j}}{\widetilde{S}_{i j} C_{i j k l}^{(E)} \widetilde{S}_{k l}+\frac{2}{3} \sigma_{y}^{2}\left(\widetilde{C}+E_{T}\right)},
$$

is a component of a stress tensor,

$$
A=\frac{2}{3} \sigma_{y} \frac{\partial \sigma_{y}}{\partial \dot{\varepsilon}_{e q}^{(V P)}} \Delta \dot{\varepsilon}_{e q}^{(V P)},
$$

is a positive scalar variable, $\Delta \sigma_{i j}$ is the increment component of the second Piola-Kirchhoff stress tensor, $D_{i j k l}^{(E)}$ are the components of tensor $\boldsymbol{D}^{(E)}=\left[\boldsymbol{C}^{(E)}\right]^{-1}$ in time $\mathrm{t}, \Delta \varepsilon_{\mathrm{ij}}$ is the increment component of Green-Lagrange strain tensor, $C_{i j k l}^{(E)}$ are the components of elastic constitutive tensor $C^{(E)}$.

\subsection{Incremental model of motion and deformation}

In this section we develop the equation of a motion and deformation of the object in the updated Lagrangian formulation. Assuming that numerical solutions are obtained at discrete time $t$, the solution for $t+\Delta t$ is to be obtained. Using the conditions of stationary of functional $\Delta \boldsymbol{J}\left(\Delta \ddot{u}_{i}, \Delta \dot{u}_{i}, \Delta u_{i}\right)=\Delta \boldsymbol{J}(\cdot)$ where $\Delta u_{i}, \Delta \dot{u}_{i}, \Delta \ddot{u}_{i}$ are the ith increment components of the displacement, velocity and acceleration vectors, respectively and a finite element method, we can write an equation of motion and deformation in the form:

$$
[\boldsymbol{M}]\{\Delta \ddot{\boldsymbol{r}}\}+\left[\boldsymbol{C}_{T}\right]\{\Delta \dot{\boldsymbol{r}}\}+\left(\left[\boldsymbol{K}_{T}\right]+\left[\Delta \boldsymbol{K}_{T}\right]\right)\{\Delta \boldsymbol{r}\}=\{\Delta \boldsymbol{R}\}+\{\Delta \boldsymbol{F}\}+\{\boldsymbol{F}\}+\{\boldsymbol{R}\},
$$

where mass matrix $[\boldsymbol{M}]$, damping matrix $\left[\boldsymbol{C}_{T}\right]$, stiffness matrix $\left[\boldsymbol{K}_{T}\right]$ and force vector $\left\{\boldsymbol{F}_{T}\right\}$ are known at time t. However, increment stiffness matrix $\left[\Delta \boldsymbol{K}_{T}\right]$, external incremental load vector $\{\Delta \boldsymbol{R}\}$, internal incremental forces vector $\{\Delta \boldsymbol{F}\}$, incremental vectors of displacement $\{\Delta \boldsymbol{r}\}$, velocity $\{\Delta \dot{\boldsymbol{r}}\}$ and acceleration $\{\Delta \ddot{\boldsymbol{r}}\}$ of finite element assembly at a typical step time are not known. In order to solve this problem we apply the integration methods - central difference method (DEM), which is one of the methods of direct integration of the equation (7).

\section{DEM solution}

Assuming that an increase of temporary step $\Delta t$ is very small, it is possible to execute a linearization of equation (7) and using the incremental decomposition we obtain:

$$
[\boldsymbol{M}]\left\{{ }^{t} \ddot{\boldsymbol{r}}\right\}+\left[\boldsymbol{C}_{T}\right]\left\{{ }^{t} \dot{\boldsymbol{r}}\right\}+\left[\boldsymbol{K}_{T}\right]\left\{{ }^{t} \boldsymbol{r}\right\}=\left\{{ }^{t} \boldsymbol{F}_{T}\right\}+\left\{{ }^{t} \boldsymbol{Q}\right\} .
$$

Then using the central difference method (DEM), in which it is assumed that: 


$$
\left.\left\{{ }^{t} \dot{\boldsymbol{r}}\right\}=0,5\left({ }^{t+\Delta t} \boldsymbol{r}\right\}-\left\{{ }^{t-\Delta t} \boldsymbol{r}\right\}\right) / \Delta t,\left\{{ }^{t} \ddot{\boldsymbol{r}}\right\}=\left(\left\{^{t+\Delta t} \boldsymbol{r}\right\}-2\left\{{ }^{t} \boldsymbol{r}\right\}+\left\{{ }^{t-\Delta t} \boldsymbol{r}\right\}\right) / \Delta t^{2}
$$

and substituting the relations in (9) into (8) we obtain:

$$
[\widetilde{\boldsymbol{M}}]\left\{{ }^{\tau} \boldsymbol{r}\right\}=\left\{{ }^{t} \widetilde{\boldsymbol{Q}}_{T}\right\},
$$

where:

$$
\begin{gathered}
{[\tilde{\boldsymbol{M}}]=[\boldsymbol{M}] / \Delta t^{2}+0,5\left[\boldsymbol{C}_{T}\right] / \Delta t,} \\
\left\{{ }^{t} \widetilde{\boldsymbol{Q}}_{T}\right\}=\left\{{ }^{t} \boldsymbol{F}_{T}\right\}+\left\{{ }^{t} \boldsymbol{Q}\right\}-\left[\boldsymbol{K}_{T}\right]\left\{{ }^{t} \boldsymbol{r}\right\}+\frac{\left.\left.2{ }^{t}{ }^{t} \boldsymbol{r}\right\}-t^{t-\Delta t} \boldsymbol{r}\right\}}{\Delta t^{2}}[\boldsymbol{M}]+\frac{\left\{^{t-\Delta t} \boldsymbol{r}\right\}}{2 \Delta t}\left[\boldsymbol{C}_{T}\right] .
\end{gathered}
$$

The integration method requires that the time step $\Delta t$ is smaller than critical value $\Delta t_{k r}$, which can be calculated from the mass and stiffness properties of the complete element assemblage: $\Delta t \leq \Delta t_{k r}=T_{N} / \pi$, where $T_{N}$ is the smallest period of the finite element assemblage with $\mathrm{N}$ degrees of freedom.

\section{Model investigation}

The model investigation was conducted in order to settle the course deformation layer top sample executed from the plastic material, as well as with the aim to qualify boundary conditions for displacements indispensable to numeric analysis of the round tread rolling process.

To improve accuracy in elaboration of the displacement vector component of the node, the real part of the thread was substituted by a rectangular model with proper magnification. The model dimension and dimension of the rectangular grid on the model satisfy the criterions of the geometrical similarity, however the material model (plasticine) and real material (steel C55) were criterions of the physical similarity.

Two samples were joined by sides with a plot mesh, and were closed in a metal form. Then the samples were subjected to the deformation by a perpendicular shift of rectilinear motion in the model stamp of an outline of round thread (fig. 3). The exemplary view of deformed samples with finite element grid for three causes of friction coefficient is presented in fig. 4.

On figure 4 observe that increasing the value of the friction coefficient on the contact surface between tool - work pieces causes an increase in the adhesion zone and decrease of material sliding on the contact surface. That has an influence on curving vertical lines of grid to the bottom of the thread. This curving is improved together with an increase in the friction coefficient.

\section{Numerical simulation of state of displacement, strain and stress of material during round thread rolling}

The application developed with regard to the method of finite elements in ANSYS program provides a complex time analysis of physical phenomena 
during the rolling process. Digital computing for the process was carried out with the use of two methods. The first method requires introducing the boundary conditions for displacements in the contact zone determined by the model investigation, whereas the second one requires the adequate determination of the contact zone without an introduction of boundary conditions. The main aim of the simulation was to define the influence of friction coefficient on the state of deformation (displacements and strain) and stress in the surface layer of the object. The numerical analysis for 2-D states of deformation and 3-D states of stress was applied on the example of steel C55 (DIN) (Fig. 5). The stand is a rigid body $E \rightarrow \infty$, however the material model as an elsto/visco-plastic body with non-linear hardening. The model has discretized by finite element PLANE183 with nonlinear function of the shape. In the second calculating method the contact tool with work pieces was modeling by element TARGE169 and CONTA171. Computational model contain 213266 degrees of freedom.

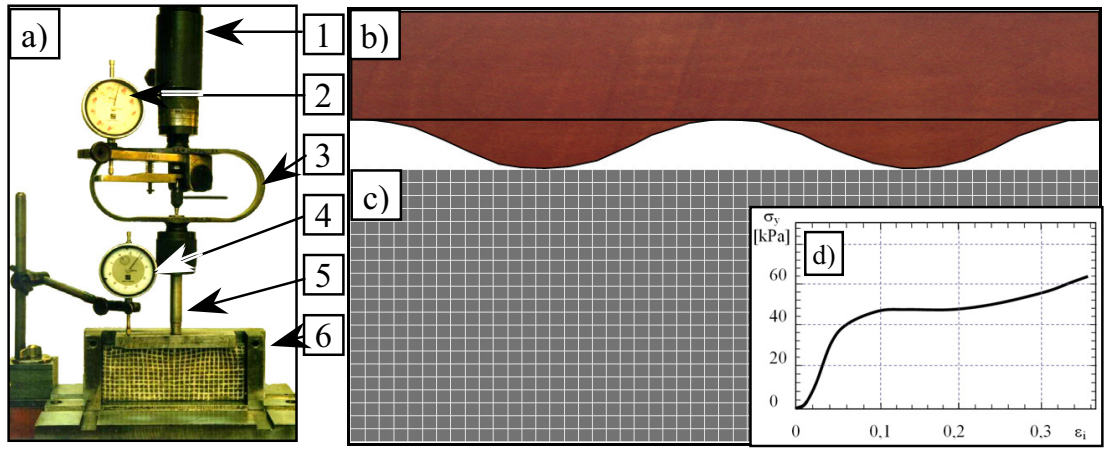

Figure 3: The view of the stand for model investigation (a), the outline of used stamp (b) and the meshed sample before deformation (c), the stress-strain curve for the material model (d): 1 - remote control hydraulic cylinder, 2 - dial gauge for force pressure on stamp, 3 dynamometer, 4 - dial gauge for measure displacement, 5 - stamp, 6 - container.
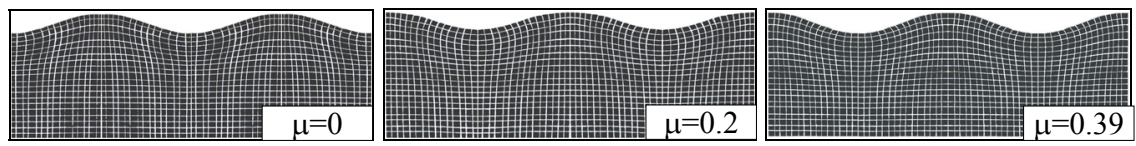

Figure 4: The mesh after deformation for $\mu=0$ (a), $\mu=0,2$ (b) and $\mu=0,39$ (c).

Exemplary results of the numerical simulation are present on figures 6 and 7. Analyzing the intensity distribution of strain, stress and deformation of the finite element grid, where the influence of the lubrication condition is observed.

For $\mu=0$ in the contact zone tool - workpieces (fig. 6(a)), during the forming of the outline of the thread, material is not broken by the tool and slide through the contact surface. The curving of the vertical line of the finite element 
grid is invisible. On the other side, an increase in the friction coefficient causes an increase in the braking of the material. For high value of the friction coefficient (fig. 6(c)) there occurs a strong braking of material in the contact zone, also from the adhesion zone of the material. That causes higher displacements of material in the zone placed father from the contact zone. Then the line of the finite element grid are stronger curved. The results of the numerical analysis are comparable with results from model investigation presented in section 4 .

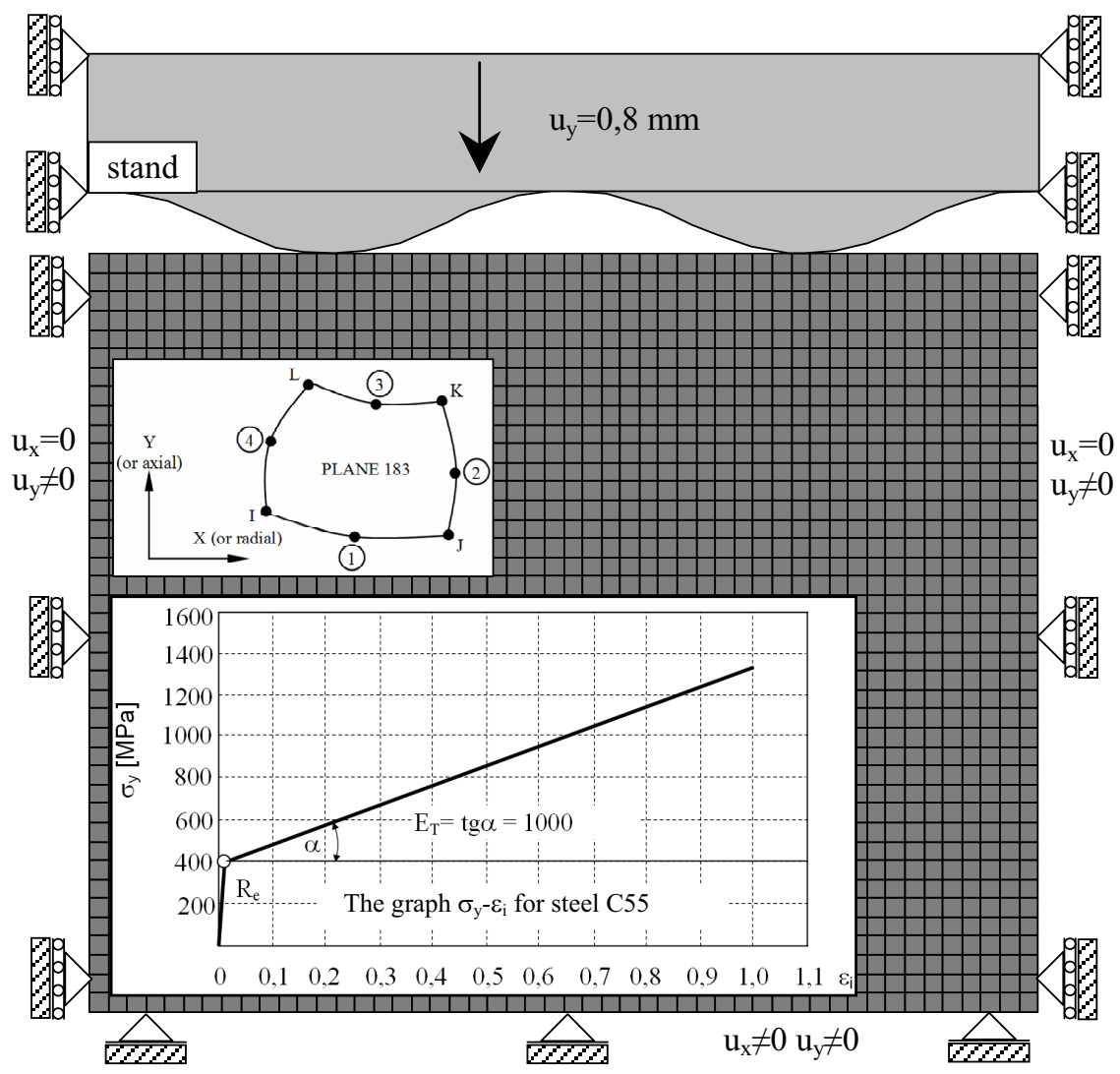

Figure 5: The discretized model with boundary conditions for displacements.

The friction coefficient also has influence on value and distribution of stress. For $\mu=0$ the maximum value of stress intensity count $\sigma_{i}=932 \mathrm{MPa}$ and is placed on the bottom of thread, for $\mu=0,39$ is less and count $\sigma_{i}=729 \mathrm{MPa}$ (MX1, Fig. $6(\mathrm{c})$ ). For $\mu>0$ appear local maximum of stress on the sides of the thread (MX2), where the value increase with increase of friction coefficient from value $\sigma_{i}=829$ MPa, for $\mu=0,2$ (Fig. 6(b)) to $\sigma_{i}=945 \mathrm{MPa}$ for $\mu=0,39$ (Fig. 6(c)). 

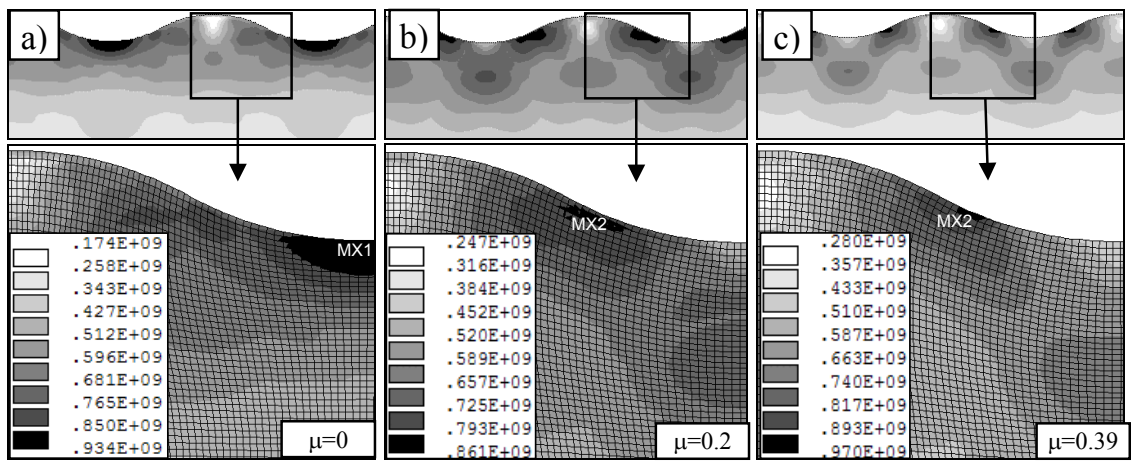

Figure 6: The deformation of grid and the stress intensity maps for various value of frictions coefficient.
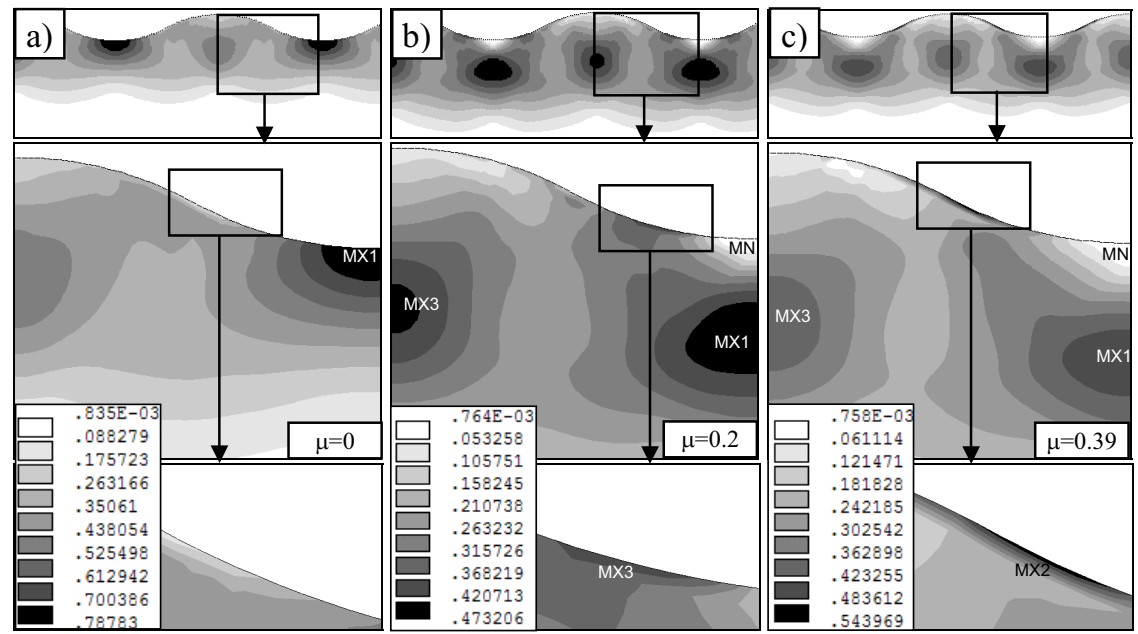

Figure 7: The strain intensity maps for various value of friction coefficient.

The friction coefficient has a high influence on value and distribution of strain. For $\mu=0$ the maximum strain intensity $\varepsilon_{i}=0,78$ is located on the bottom of the thread, close to the contact surface (MX1, Fig. 7(a)). For $\mu>0$ appear an adhesion zone of material in the bottom of the thread, which take the characteristic shape of a wedge. In this zone the value of strain is very small. For $\mu=0,2-\varepsilon_{i}=0,1 \quad(\mathrm{MN}$, Fig. 7(b)) and for $\mu=0,39$ strains are closer to the contact surface and getting smaller to value $\varepsilon_{i}=0,0016$ (elastic strains) (MN, Fig. 7(c)). However, the local maximum strains (MN, Fig. 7(c)) are more and more moving down from the contact surface. Two local maximums of the strains appear. The first one (MX2) is placed close to the contact zone of the side of the thread, where a higher value of friction coefficient increase strains value from $\varepsilon_{\mathrm{i}}=0,4$ for $\mu=0,2$ (Fig. $7(\mathrm{~b})$ ) to value $\varepsilon_{i}=0,54$ for $\mu=0,39$. The next one, 
local maximum (MX3), is located in the depth of material on a symmetry axis that passes through the top of the thread. Here, strains are getting smaller together with increasing of the friction coefficient from value $\varepsilon_{i}=0,44$ for $\mu=0,2$ (fig. 7(b)) to $\varepsilon_{i}=0,42$ for $\mu=0,39$ (fig. 7(c)).

\section{Conclusions}

The round thread rolling process on the pipes is a geometrical, physical and thermal non-linear and boundary problem. Measurement of the process parameters that influence the technological quality, such as: a displacement zone, a temperature, stress, structural change etc. during the thread rolling process is impossible without today's modern techniques of a measurement.

An application of modern numerical methods and computing systems allows an analysis of complex physical phenomena occurring in the process under investigation. The application developed in the ANSYS system enables a time analysis of the rolling process with the consideration of the changeability of the lubrications conditions. On the course of physical phenomena in the working zone we can forecast a technological quality of the round thread.

The results obtained of the computer simulation of the thread rolling process show that the friction coefficient influence the states of displacements, strains and stresses in the surface layer of the thread, also that it is one of the factors affecting the technological and the exploitation quality. The best operational quality of the thread is received during the rolling process with high lubrication conditions $(\mu=0)$.

The simulation results for the condition of lubrication can be of use while designing the round thread rolling process: making a selection of the process condition and the kind of lubrication factor in the aspect of the technological quality of the screw.

\section{References}

[1] Łyczko K.: The technology of tool and the female thread rolling. Częstochowa University of Technology, 1999 (in polish).

[2] Kukielka L., Kukielka K.: Numerical analysis of the process of trapezoidal thread rolling, III International Conference on High Performance Structures and Materials, 3-5 May 2006, Ostand, Belgium. WITPRESS Southampton, Boston, 2006, pp. 663-672.

[3] Kukielka K., Kukielka L.: Modeling And Numerical Analysis Of The Thread Rolling Process, 77th Annual Meeting of the Gesellschaft für Angewandte Mathematik und Mechanik, March 27th - 31st, Technische Universität Berlin, 2006. 